\title{
Critical behavior of a fluid in a disordered porous matrix. An Ornstein-Zernike approach.
}

\author{
E. Pitard ${ }^{1}$, M.L. Rosinberg ${ }^{1}$, G. Stell ${ }^{2}$ and G. Tarjus ${ }^{1}$ \\ ${ }^{1}$ Laboratoire de Physique Théorique des Liquide\$, Université Pierre et Marie Curie, \\ 4 Place Jussieu, 75252 Paris Cedex 05, France \\ ${ }^{2}$ Department of Chemistry, State University of New York at Stony Brook, \\ Stony Brook, NY 11794, USA
}

(August 15, 2018)

\begin{abstract}
Using a liquid-state approach based on Ornstein-Zernike equations, we study the behavior of a fluid inside a porous disordered matrix near the liquid-gas critical point. The results obtained within various standard approximation schemes such as lowest-order $\gamma$-ordering and the mean-spherical approximation suggest that the critical behavior is closely related to that of the randomfield Ising model (RFIM).
\end{abstract}

Pacs numbers: 05.70.Fh, 61.20.Gy, 64.60.Fr

Typeset using REVTEX 
The interpretation of experimental studies of the phase behavior of fluids (binary mixtures [1] or ${ }^{4} \mathrm{He}$ [2]) adsorbed in disordered porous materials such as silica gels or Vycor remains highly controversial. Some results lend support to the view that preferential adsorption of one component and randomness of the pore network give rise to a random-field Ising-like behavior near the critical point, as suggested by Brochard and de Gennes [3]. On the other hand, it has been argued that the experiments in Vycor, which is a glass with a fairly low porosity, can be interpreted in terms of wetting in a confined geometry, with no randomness [4]. Clear-cut conclusions are somewhat obscured by the metastable and non-equilibrium effects which are often present in the experiments. However, there is a need for more realistic continuum descriptions which encompass both randomness, confinement, and connectivity between pores. The goal of this letter is to present a liquid-state approach describing a single component fluid in a rigid disordered matrix and, starting from this microscopic theory, to give some insight on the critical properties of the fluid and their relation to the random-field Ising model (RFIM) [5].

Following the formalism recently proposed by Madden and Glandt [6], we consider the matrix-fluid system as a special binary mixture in which the matrix is treated as a rigid set of obstacles, obtained by quenching an equilibrium configuration of particles (species 0), with the fluid particles (species 1) at equilibrium in the presence of (and in interaction with) the quenched particles. The structure of this quenched-annealed (Q-A) mixture is described by the total pair correlation functions $h_{i j}(r)$ which satisfy a set of coupled integral equations, the so-called replica Ornstein-Zernike (ROZ) equations [7]. As in the usual OZ formalism [8], these equations relate the functions $h_{i j}(r)$ to the corresponding direct correlation functions $c_{i j}(r)$ which are expected to be short-ranged (i.e, $\int d^{d} \mathbf{r} c_{i j}(r)$ is finite), even at the critical point. The exact form of the ROZ equations can be most easily derived by using the replica trick which replaces the study of the original system by that of an equilibrium mixture composed of $s$ copies of the fluid interacting with the matrix. It should be stressed that, in contrast to the standard replica treatment of quenched disorder, there is no direct interaction between the fluid replicas because the average over disorder (i.e., over all possible 
configurations of the matrix particles) is not performed explicitly. These ROZ equations represent the starting point of our treatment and their formal solution in Fourier space is given by

$$
\begin{gathered}
\hat{h}_{00}(k)=\frac{\hat{c}_{00}(k)}{1-\rho_{0} \hat{c}_{00}(k)} \\
\hat{h}_{01}(k)=\frac{\hat{c}_{01}(k)}{\left[1-\rho_{0} \hat{c}_{00}(k)\right]\left[1-\rho_{1} \hat{c}_{c}(k)\right]} \\
\hat{h}_{c}(k)=\frac{\hat{c}_{c}(k)}{1-\rho_{1} \hat{c}_{c}(k)} \\
\hat{h}_{b}(k)=\left[\hat{c}_{b}(k)+\rho_{0} \frac{\hat{c}_{01}^{2}(k)}{1-\rho_{0} \hat{c}_{00}(k)}\right] \frac{1}{\left[1-\rho_{1} \hat{c}_{c}(k)\right]^{2}}
\end{gathered}
$$

where $\hat{f}(\mathbf{k})$ denotes the Fourier transform of a function $f(\mathbf{r})$ and $\rho_{i}$ is the average density of species $i$. In deriving these equations, it has been assumed that there is no replica symmetry breaking. As in the case of disordered magnetic systems, one has introduced the connected part, $h_{c}(r)$, and the blocked or disconnected part, $h_{b}(r)=h_{11}(r)-h_{c}(r)$, of the fluid-fluid correlation function [7,9]; they are equal, respectively, to the $s=0$ limits of $h_{\alpha \alpha}(r)-h_{\alpha \beta}(r)$ and $h_{\alpha \beta}(r)$, where $\alpha$ and $\beta \neq \alpha$ denote fluid replicas. Similarly, $c_{c}(r)$ and $c_{b}(r)$ are defined as the connected and blocked parts of $c_{11}(r)$. By symmetry, one has $h_{01}=h_{10}$ and $c_{01}=c_{10}$.

The above ROZ equations differ from those recently derived for a fluid in presence of a quenched, random, external potential obeying a Gaussian distribution [10]: in Eq. (1d), the quenched disorder enters explicitly through a term involving the matrix-matrix and matrix-fluid direct correlation functions which also appear in Eqs. (1a) and (1b), whereas in Ref. [10] it is only contained in the inter-replica pair potential. As a result, the present formulation is also able to take into account the geometrical constraint on the fluid due to the presence of the matrix (excluded volume and/or confinement effects).

It has been shown [9, 11] that $\hat{c}_{c}(0)$ is related to $\chi_{1}$, the compressibility of the fluid inside the matrix (i.e., the derivative of the fluid pressure $P_{1}$ with respect to the density or, 
through the Gibbs-Duhem equation [9], the derivative of the fluid chemical potential), via the equation

$$
k_{B} T \rho_{1} \chi_{1}=\left[1-\rho_{1} \hat{c}_{c}(0)\right]^{-1}
$$

Therefore, the gas-liquid critical point of the fluid inside the matrix, if it exists, is characterized as usual by both a diverging compressibility and long-range correlations. However, a special feature of Q-A systems is that the disconnected part of the fluid-fuid correlation function, and as a consequence $h_{11}(r)$ itself, may be much longer-ranged that the connected part. Indeed, if one defines the exponents $\eta$ and $\bar{\eta}$ by $h_{c}(r) \sim r^{-d+2-\eta}$ and $h_{b}(r) \sim r^{-d+4-\bar{\eta}}$ when $r \rightarrow \infty$ at the critical point, one finds immediately from Eqs. (1c) and (1d) that $\bar{\eta}=2 \eta$. This result rests only on the assumption that the matrix is non-critical, i.e., $1-\rho_{0} \hat{c}_{00}(0) \neq 0$, and that $\hat{c}_{b}(0)$ and $\hat{c}_{01}(0)$ are finite, a condition generally expected for direct correlation functions. This relation between $\bar{\eta}$ and $\eta$ is for instance satisfied within the standard Ornstein-Zernike approximation which assumes that the direct correlation functions have the same range as the associated pair interactions. For short-ranged interactions, this gives $\bar{\eta}=\eta=0$, but for long-ranged fluid-fluid interactions decaying as $r^{-(d+\sigma)}$ for $r \rightarrow \infty$ with $\sigma \leq 2$, one obtains $\bar{\eta}=4-2 \sigma=2 \eta$. Note that the same relation $\bar{\eta}=2 \eta$ has been proposed for the RFIM [12] and seems to be confirmed by recent numerical estimates [13.

To be more specific, we now suppose that the fluid-fluid (resp. matrix-fluid) pair potential can be divided into a reference part which includes the short-range repulsive part of the interaction and a more smoothly varying long-range attractive part $w_{11}(r)$ (resp. $\left.w_{01}(r)\right)$. As a first step, we derive the mean-field (MF) results. As in the bulk fluid case, this can be done by considering the lowest $\gamma$ - ordering approximation which is derived by introducing the inverse range parameter $\gamma$ in the attractive fluid-fluid potential, $w_{11}(r)=\gamma^{d} \phi(\gamma r)$, and then taking the limit $\gamma \rightarrow 0$ (see for instance Ref. [8]) . From the graphical expansion of the three direct correlation functions $c_{01}(r), c_{b}(r)$ and $c_{c}(r)$ [6], it is easy to show that $\hat{c}_{01}(k)=\hat{c}_{01}^{R}(k)$, $\hat{c}_{b}(k)=\hat{c}_{b}^{R}(k)$ and $\hat{c}_{c}(k)=\hat{c}_{c}^{R}(k)-\left(k_{B} T\right)^{-1} \hat{\phi}(0) \delta_{k, 0}$, where $\delta_{k, 0}$ is the Kronecker symbol and 
the superscript $R$ indicates a quantity calculated in a reference system for which $w_{11}(r) \equiv 0$. The fact that $\hat{c}_{b}$ reduces to its reference part illustrates the fact that the fluid replicas are not directly coupled (see above). Using Eq. (2), we immediately obtain a van der Waals equation of state, $P_{1}=P_{1}^{(R)}+\frac{1}{2} \rho_{1}^{2} \hat{\phi}(0)$. From the thermodynamic relations satisfied by the Q-A mixture [9], we can conclude that besides $\eta=\bar{\eta}=0$, the critical behavior is described by the usual MF exponents, $\nu=1 / 2, \alpha=0, \beta=1 / 2, \gamma=1, \delta=3$. As illustrated numerically in Ref. [14 for a specific system, the critical point is displaced from the MF critical point of the bulk fluid because the reference system includes the influence of the host matrix (note that in Ref. [14 the matrix-fluid interaction is also treated in the MF approximation).

To go further, some more quantitative statement on the correlations over small distances must be made. This can be implemented, within the $\mathrm{OZ}$ approximation mentioned above, by considering approximate closure relations to the $\mathrm{ROZ}$ equations like the mean-spherical approximation (MSA) [8]. For bulk fluids, the MSA is known to yield the same critical exponents as the spherical spin model [15]. We now show that the same is true for a Q-A system. To avoid unnecessary technical difficulties, we consider the case of a d-dimensional lattice gas. We expect that the behavior in the critical region will be identical to that of a real continuum fluid. Lattice cells (or sites) can be occupied either by matrix or fluid "particles" and the pair potentials contain a " hard-core" part which excludes multiple occupancy of a cell (or site). It must be noted that even for the lattice fluid there is no holeparticle symmetry because of the interaction with the matrix. Therefore, there is no exact correspondence between this microscopic description of a fluid inside a matrix and the usual RFIM (see also Ref. [16] for a related discussion). More precisely, the standard equivalence between the lattice-gas and the spin $1 / 2$ model shows that we are actually dealing with a site diluted Ising model where the spins are coupled to correlated external random fields which depend on the site disorder variables [17]. Application of the MSA closure in the matrix-replicas mixture (i.e. $k_{B} T c_{\alpha \beta}(\mathbf{r})=-w_{\alpha \beta}(\mathbf{r})$ for $\mathbf{r} \neq 0$ ) gives, in the limit $s \rightarrow 0$, 


$$
\begin{aligned}
c_{00}(\mathbf{r}) & =-\frac{\delta_{\mathbf{r}, 0}}{\left(1-\rho_{0}\right)}, \quad c_{01}(\mathbf{r})=c_{01}^{0} \delta_{\mathbf{r}, 0}-\beta w_{01}(\mathbf{r}) \\
c_{b}(\mathbf{r}) & =0, \quad c_{c}(\mathbf{r})=c_{c}^{0} \delta_{\mathbf{r}, 0}-\beta w_{11}(\mathbf{r})
\end{aligned}
$$

where $c_{01}^{0}$ and $c_{c}^{0}$ are functions of temperature and fluid density, which, owing to the structure of the ROZ equations, are completely determined by imposing the "core" conditions $h_{01}(\mathbf{r}=$ $0)=h_{11}(\mathbf{r}=0)=-1$. Note that $h_{c}$ and $h_{b}$ do not satisfy themselves the core requirement. On the other hand, the prescription for $c_{00}$ is the direct consequence of the core condition for the matrix particles, $h_{00}(\mathbf{r}=0)=-1$ (for simplicity, we assume that there is no attractive interaction between matrix particles).

For clarity, we first consider nearest-neighbor (n.n.) interactions in a hypercubic lattice. Generalizing the analysis developped by Stell [15] and Theumann [18] for the bulk lattice-gas, we write the inverse Fourier transform of $\hat{\chi}(\mathbf{k})=1+\rho_{1} \hat{h}_{c}(\mathbf{k})$ as

$$
-\frac{\rho_{1} w_{11} \chi(\mathbf{r})}{k_{B} T} \equiv G\left(\mathbf{r}, \kappa^{2}\right)=\int_{-\pi}^{\pi} \frac{d^{d} \mathbf{k}}{(2 \pi)^{d}} \frac{e^{i \mathbf{k} . \mathbf{r}}}{\kappa^{2}+2 \sum_{j=1}^{d}\left(1-\cos k_{j}\right)}
$$

where $\mathbf{k}=\left(k_{1}, \ldots, k_{d}\right)$ and $\kappa=\left[-2 d+k_{B} T\left(1-\rho_{1} c_{c}^{0}\right) /\left(-\rho_{1} w_{11}\right)\right]^{1 / 2}$ is an inverse correlation length which vanishes at the critical point. By replacing in the ROZ equations, we find

$$
\frac{-\rho_{1} w_{11}}{\left(1-\rho_{0}\right)} h_{01}(\mathbf{r})=f G\left(\mathbf{r}, \kappa^{2}\right)+w_{01} \delta_{\mathbf{r}, 0}
$$

and

$$
\frac{\left(\rho_{1} w_{11}\right)^{2}}{\rho_{0}\left(1-\rho_{0}\right)} h_{b}(\mathbf{r})=w_{01}^{2}+2 w_{01} f G\left(\mathbf{r}, \kappa^{2}\right)-f^{2} G^{\prime}\left(\mathbf{r}, \kappa^{2}\right)
$$

where $G^{\prime}\left(\mathbf{r}, \kappa^{2}\right) \equiv \partial G\left(\mathbf{r}, \kappa^{2}\right) / \partial \kappa^{2}$ and $f=k_{B} T c_{01}^{0}-w_{01}\left(\kappa^{2}+2 d\right)$. Obviously, $h_{c}(\mathbf{r}), h_{b}(\mathbf{r})$ and $h_{01}(\mathbf{r})$ have the same correlation length $\kappa^{-1}$, and $\eta=\bar{\eta}=0$. If $d \leq 4$, no finite critical temperature exists since this would lead to the unacceptable result that $h_{b}(\mathbf{r})$ and thus $h_{11}(\mathbf{r})$ do not decay to zero when $r \rightarrow \infty$ at the critical point. Using the core conditions together with Eqs. (4-6) yields an implicit equation relating $\kappa$ to the thermodynamic variables. In absence of hole-particle symmetry, the critical point is found by requiring that $\left(\partial P_{1} / \partial \rho_{1}\right)_{c}=$ $\left(\partial^{2} P_{1} / \partial \rho_{1}^{2}\right)_{c}=0$ on the critical isotherm, which from Eqs. (2) and (4) is equivalent to 
$\kappa_{c}=\left(\partial \kappa^{2} / \partial \rho_{1}\right)_{c}=0$. For our system, this yields $\rho_{1 c}=\left(1-\rho_{0}\right)\left(1+2 y q \rho_{0}\right) / 2\left(1+q \rho_{0}\right)$ with $q=-1-G^{\prime}(0,0) / G^{2}(0,0) \geq 0$ and $y=w_{01} / w_{11}$, whereas the shift of the critical temperature due to the presence of the matrix is given by $T_{c} / T_{c}^{b u l k}=\left(1-\rho_{0}\right)\left[1+4 y(1-y) q \rho_{0}\right] /\left[1+q \rho_{0}\right]$; this ratio being always smaller than 1 , one has $T_{c} \leq T_{c}^{b u l k}$. The behavior of the correlation length in the critical regime can now be obtained by expanding the implicit equation for $\kappa$ in terms of $\Delta T=T-T_{c}, \Delta \rho_{1}=\rho_{1}-\rho_{1 c}$, and $\kappa$. Using the asymptotic expansion of the Green function $G\left(\mathbf{r}=0, \kappa^{2}\right)$ [15], we finally obtain for $d>4$

$$
\begin{aligned}
-\frac{G(0,0)}{w_{11}} k_{B} \Delta T & +\frac{1+q \rho_{0}}{1-\rho_{0}}\left(\Delta \rho_{1}\right)^{2}=k_{B} T_{c} \frac{G^{\prime}(0,0)}{w_{11}} \kappa^{2} \\
& +\frac{\rho_{0}\left(1-\rho_{0}\right)}{4\left(1+q \rho_{0}\right)^{2}}(1-2 y)^{2}\left[A \kappa^{2}+B \kappa^{d-4}+C \kappa^{d-4} \ln \kappa+\ldots\right]
\end{aligned}
$$

where A, B are nonzero constants and $C=0$ when and only when $\mathrm{d}$ is odd. From this we can read off the exponents of the correlation length along the critical isochore $(\nu)$ and the critical isotherm ( $\epsilon$, in the notation of Ref. [15]), and then, with the help of the compressibility equation (2) and the Gibbs-Duhem relation [9], the values of $\gamma$ and $\delta$. For $d \geq 6$, one recovers the MF exponents whereas for $4<d \leq 6$ one has $\nu=1 /(d-4), \epsilon=2 /(d-4)$, $\gamma=2 /(d-4)$ and $\delta=d /(d-4)$. On the other hand, when $y=1 / 2$, one finds the MF exponents as soon as $d \geq 4$. In this latter case, the system is easily shown to be isomorphic to the site diluted spin model with no random fields.

Using the expression of the configurational internal energy, $U_{1}=1 / 2 \rho_{1} \hat{w}_{11}(0)+\rho_{0} \hat{w}_{10}(0)+$ $1 / 2 \sum_{\mathbf{r} \neq 0}\left[\rho_{1} w_{11}(\mathbf{r}) h_{11}(\mathbf{r})+2 \rho_{0} w_{10}(\mathbf{r}) h_{10}(\mathbf{r})\right]$, and deriving with respect to temperature, one finds that the critical exponent $\alpha$ of the fluid specific heat $C_{1 v}$ is always zero whereas the exponent $\alpha_{s}$ characterizing the singular part of $C_{1 v}$, if present, is always negative and given by $\alpha_{s}=(d-6) /(d-4)$ for $4<d<6$ and $\alpha_{s}=(6-d) / 2$ for $d \geq 6$. Finally, the same arguments as for the bulk lattice-gas [15] lead to $\beta=1 / 2$ for the coexistence curve exponent. These calculations can be easily generalized to non n.n. interactions and to cases where $w_{01}(r)$ and $w_{11}(r)$ do not have the same range. The same exponents are recovered except that when $w_{11}(r)$ dies off like $r^{-(d+\sigma)}$ with $\sigma<2$ one finds $\nu=1 /(d-2 \sigma), \gamma=\sigma /(d-2 \sigma)$, $\delta=d /(d-2 \sigma)$ and $\alpha_{s}=(d-3 \sigma) /(d-2 \sigma)$ for $2 \sigma<d<3 \sigma$, whereas $\nu=1 / \sigma, \gamma=1, \delta=3$ 
and $\alpha_{s}=(3 \sigma-d) / \sigma$ for $d \geq 3 \sigma$.

Except for the special case which reduces to the site diluted spin model without random field, all these exponents coincide with those of the spherical model in a random field [19]: in particular, we find that the disorder induces a dimensional shift $d \rightarrow d-2$ for short-range interactions and $d \rightarrow d-\sigma$ for long-range interactions. These results are valid even in the absence of attractive matrix-fluid interaction ( $y=0$ in the case of n.n interactions). The fact that the Q-A system and the random-field spin model have the same behavior in the critical region does not garantee, however, that their thermodynamics coincide, and a more complete study of the MSA phase diagram will be presented elsewhere [17].

To summarize, we have related the critical behavior of a fluid in a porous matrix to that of the RFIM by using common approximations of liquid-state theory [20]. The present treatment based on the ROZ equations takes into account both disorder and excluded volume effects. The fact that the connection with the RFIM holds also in the absence of matrix-fluid attractive interactions suggests that preferential adsorption may not be a crucial ingredient for observing a random-field like behavior. Of course, the identity of the critical exponents within various approximation schemes is not a proof that the two systems belong to the same universality class [21]. To go further, one must implement the techniques of the renormalization group, which can be considered in connection with liquid-state theory [22]. One must also investigate a possible replica symmetry breaking (RSB) mechanism which seems to occur in the RFIM [23] (however, this is not expected at the MF [24] or sphericalmodel levels [23]). For that purpose, it is necessary to use a non-linear closure to the ROZ equations, such as the hypervertex approximations discussed in Ref. [25]. If RSB indeed occurs in Q-A systems, the assumption that the direct correlation functions remain shortranged, even in the critical region, may lead to non trivial predictions.

G. S. gratefully acknowledges the support of the National Science Foundation. 


\section{REFERENCES}

* $\quad$ Unité de Recherche Associée au CNRS (URA 765).

[1] M.C. Goh, W. I. Goldburg, and C. Knobler, Phys. Rev. Lett. 58, 1008 (1987); S. B. Dierker and P. Wiltzius, ibid. 58, 1865 (1987); S. B. Dierker and P. Wiltzius, ibid. 66, 1185 (1991); B. J. Frisken and D. S. Canell, ibid. 69, 632 (1992).

[2] A. P. Y. Wong and M. H. W. Chan, Phys. Rev. Lett. 65, 2567 (1990).

[3] F. Brochard and P. G. de Gennes, J. Phys. Lett. (Paris) 44, 785 (1983); P. G. de Gennes, J. Phys. Chem. 88, 6469 (1984).

[4] L. Monette, A. Liu and G. S. Grest, Phys. Rev. A 46, 7664 (1992).

[5] for recent reviews see T. Nattermann and J. Villain, Phase Transitions, 11, 817 (1988); D. P. Belanger and A. P. Young, J. Magn. \& Magn. Mater. 100, 272 (1991).

[6] W. G. Madden and E. D. Glandt, J. Stat. Phys. 51, 537 (1988).

[7] J. A. Given and G. Stell, J. Chem. Phys. 97, 4573 (1992); E. Lomba, J. A. Given, G. Stell, J.J. Weis, and D. Levesque, Phys. Rev. E 48, 223 (1993).

[8] J. P. Hansen and I. R. McDonald, Theory of Simple Liquids (Academic, New York, 1976).

[9] M. L. Rosinberg, G. Tarjus, and G. Stell, J. Chem. Phys. 100, 5172 (1994).

[10] G. I. Menon and C. Dagupta, Phys. Rev. Lett. 73, 1023 (1994).

[11] D. M. Ford and E. Glandt, J. Chem. Phys. 100, 2391 (1994).

[12] M. Schwartz, J. Phys. C 18, 135 (1985).

[13] M. Gofman, J. Adler, A. Aharony, A. B. Harris, and M. Schwartz, Phys. Rev. Lett. 71, 1569 (1993).

[14] D. M. Ford and E. Glandt, Phys. Rev. E 50, 1280 (1994). 
[15] G. Stell, Phys. Rev. 184, 135 (1969); G. Stell, in Phase transitions and critical phenomena, edited by C. Domb and M. S. Green (Academic, London, 1976), Vol. 5b, p. 205.

[16] D. Stauffer and R. B. Pandey, J. Phys. A 25, L1079 (1992).

[17] E. Pitard, M. L. Rosinberg, G. Stell, and G. Tarjus, in preparation.

[18] W. K. Theumann, Phys. Rev. B 2, 1396 (1970).

[19] P. Lacour-Gayet and G. Toulouse, J. Phys. (Paris), 35, 425 (1974).

[20] We have also calculated the critical behavior of the Q-A mixture within the generalized mean spherical approximation (GMSA) in its self-consistent version (J. S. Hoye and G. Stell, Mol. Phys. 52, 1071 (1984)): one then recovers the exponents of the Gaussian model of spins in a random field [19].

[21] However, since the usual thermodynamic relations remain valid in a Q-A system [9], one can repeat Widom's scaling approach of the thermodynamic potentials in terms of the reduced fluid variables to get $\alpha+2 \beta+\gamma=2, \gamma=\beta(\delta-1)$, as well as $\gamma=(2-\eta) \nu$. Moreover, a naive scaling ansatz for the disconnected part of the fluid-fluid pair density in the two-phase region yields the relation $2 \beta=(d-4-\bar{\eta}) \nu$. Therefore, one has $2-\alpha=[d-(2-\bar{\eta}+\eta)] \nu$ and the usual hyperscaling relation is modified, the dimension of the system being apparently shifted by $2-\bar{\eta}+\eta$ (or $2-\eta$, if we use the relation $\bar{\eta}=2 \eta$ ). All these relations between exponents, including the modified hyperscaling relation are the same as in the RFIM [5].

[22] See e.g., A. Parola, A. Meroni and L. Reatto, Phys. Rev. Lett. 62, 2981 (1989).

[23] M. Mezard and A. P. Young, Europhys. Lett. 18, 653 (1992).

[24] T. Schneider and E. Pytte, Phys. Rev. B 15, 1519 (1977).

[25] G. Stell, J. L. Lebowitz, S. Baer, and W. Theumann, J. Math. Phys. 7, 1532 (1966). 